\title{
A Metric for Comparing the Anthropomorphic Motion Capability of Artificial Hands
}

\author{
Thomas Feix, Javier Romero, Carl Henrik Ek, Heinz-Bodo Schmiedmayer, and Danica Kragic
}

\begin{abstract}
We propose a metric for comparing the anthropomorphic motion capability of robotic and prosthetic hands. The metric is based on the evaluation of how many different postures or configurations a hand can perform by studying the reachable set of fingertip poses. To define a benchmark for comparison, we first generate data with human subjects based on an extensive grasp taxonomy. We then develop a methodology for comparison using generative, nonlinear dimensionality reduction techniques. We assess the performance of different hands with respect to the human hand and with respect to each other. The method can be used to compare other types of kinematic structures.
\end{abstract}

Index Terms-Biologically inspired robots, grasping, kinematics, multifingered hands, rehabilitation robotics.

\section{INTRODUCTION}

$\mathbf{W}$ E use our hands for daily interaction with the environment-objects with which we interact have been made to suit our dexterity. We expect robots to interact with and manipulate objects made for our dexterity. The same is desired for prosthetic hands. Consequently, we have the following question: How sophisticated should the hands we build be, and how should these be designed to fully exploit their capabilities? Historically, the road of building artificial hands has stretched between building simple industrial grippers and designing more complex hands that mimic human hand anthropomorphism and dexterity [1].

In order to achieve the latter, adding more actuators results in a higher number of independently controlled joints. However, the effective dexterity of such a hand may not be increased due to the complexity in control [2]. Thus, there is an interest in

Manuscript received January 17, 2012; revised June 5, 2012; accepted August 28, 2012. Date of publication September 27, 2012; date of current version February 1, 2013. This paper was recommended for publication by Associate Editor J. Dai and Editor W. K. Chung upon evaluation of the reviewers' comments. This work was supported by European Union (EU) IST FP7 Integrated Project: Topology Based Motion Synthesis for Dexterous Manipulation, EU European Research Council project: Flexible object manipulation through statistical learning and topological representations, and Swedish Foundation for Strategic Research.

T. Feix is with the Department of Mechanical Engineering and Materials Science, Yale University, New Haven, CT 06511 USA (e-mail: thomas@ @ief.net).

J. Romero is with Perceiving Systems Group, Max-Planck Institute for Intelligent Systems, Tübingen 72076, Germany (e-mail: javier.romero@ tuebingen.mpg.de).

C. H. Ek and D. Kragic are with the Computational Vision and Active Perception Lab, Centre for Autonomous Systems, The Royal Institute of Technology, 10044 Stockholm, Sweden (e-mail: chek@csc.kth.se; dani@kth.se).

H. B. Schmiedmayer is with Institute of Mechanics and Mechatronics, University of Technology, Vienna 1040, Austria (e-mail: heinz-bodo. schmiedmayer@tuwien.ac.at).

Color versions of one or more of the figures in this paper are available online at http://ieeexplore.ieee.org.

Digital Object Identifier 10.1109/TRO.2012.2217675 creating hands that are relatively simple in their design but still versatile in terms of the actions they can accomplish. Several studies in robotic hand design have been inspired by the human hand [3]-[5]. The relation between human and prosthetic hands is clear, given their similarity in the kinematic structure. The problem of which joints should be actuated in order to maximize the functionality of the hand, however, remains open. Thus, the natural question is how to define a metric suitable for performing the analysis of the hands' capabilities. Here, many parameters can be taken into account-ranging from kinematic and dynamic properties to the choice of material (rigid versus soft) and interaction with objects. At this time, no generally accepted metric for performing such an analysis exists. Similarly to our work, most of the approaches addressing the problem are data driven.

The approach presented in this paper is based on the definition of an anthropomorphism index (AI) that measures the similarity or overlap between the spaces of reachable fingertip poses for hands with different kinematic structures. At first, data with human subjects are generated, using the human hand as a reference. The generated human data are based on an extensive grasp taxonomy, including most static grasp types. The kinematics of hand movements are analyzed by determining the extent of the space of fingertip poses, spanned by different human grasp types, can be covered by different kinematic structures. It is, therefore, straightforward to change parameters of the hand models and determine their impact on the AI. Parameters that can be changed are, for example, the number of joints, their orientation, etc. This provides a fast way of generating and assessing changes in the hand design, providing the basis for incremental improvement.

An important contribution of the work is the employment of the nonlinear dimensionality reduction techniques for encoding the sparse high-dimensional data in a compact manner. We also contribute with the public release of the benchmark procedure through an open-source toolbox, ${ }^{1}$ allowing researchers to test, modify, and redistribute it. We survey the related work in Section II, and introduce the overall approach in Section III. In Section IV, we present the dimensionality reduction methods and in Section V, we define the metrics used for the comparison. In Sections VI and VII, we present the evaluation results and conclude the work in Section VIII.

\section{RELATED WORK}

A human hand model consists of approximately 20 independent joints [6]-[8]. This is an overrepresentation in terms of

\footnotetext{
${ }^{1}$ http://grasp.xief.net
} 
degrees of freedom (DoFs) as there are strong correlations between the joints [9]-[11]. The correlations are not obvious and cannot be modeled explicitly; therefore, data-driven approaches are commonly used to determine the coupling between them. Thus, only a few parameters suffice to define a hand posture [9] or hand movement [10]-[12]. The minimum number of parameters required to specify the posture of the hand is called the intrinsic dimension of the hand or the number of DoF of a hand.

In robotics, a significant effort has been put on creating sophisticated hands with the goal of mimicking the versatility of the human hand: the UB Hand 3 [13] with 16 DoF, the Robonaut Hand [4] with 12 DoF, and the DLR-HIT Hand II with 15 DoF [5]. These hands have a high-potential dexterity by design, but the true dexterity is much lower due to the control complexity [2]. The recent focus has shifted from complex to simpler hands that still can accomplish all assigned tasks [14].

The mechanical complexity of a hand and the complex handobject interactions make it difficult to assess the quality of a hand design without its realization. Furthermore, there is no common benchmark for grasp performance measures. The classic way of determining the quality of a grasp is to assess its stability based on form or force closure [15]. It is not practical to use such a measure as a guideline for the hand design, since a hand should be built so that it has a good "stability score" on many different objects. An elaborate comparison of different grasp similarity measures is presented in [16]. However, only a few hand prototypes are based on a structured analysis of their capabilities.

There are other relevant approaches to hand design optimization. The approach presented in [17] and [18] has been specialized toward underactuated kinematic hand setups. The actuation parameters of a hand are optimized to maximize the number of stable grasps achieved within a manually defined pool of grasping postures. In [18], a prototype of a simple two-fingered gripper was built, and for this special case, it was possible to calculate the global optimal solution. For more complex embodiments, the objective function becomes more difficult to handle, having multiple local minima. An approach considering postural synergies is presented in [19]. The number of in-hand rigid-body object motions depends on the number of synergies (defined as basis vectors of a linear subspace) driving the hand. It was shown that an increased number of synergies results in more movements and forces being controllable. The proposed analysis is a good tool for estimating how complex a hand has to be in order to achieve a desired degree of dexterity. Nevertheless, this tool is limited to linear subspace analysis and, therefore, to linear joint couplings.

A framework for testing of underactuated hands is presented in [20] and [16], where the ability of different kinematic setups to perform pinch or power grasp is assessed. The system determines the hand's ability to stably grasp moving cylindrical objects, as well as the grasp resistance to external forces. However, the system is used to evaluate a symmetrical gripper, where all axes are parallel. It is not clear how the system could be applied to more anthropomorphic hands, where the joint axes are not parallel, which is particularly important for the thumb.
Using the tendon-driven Anatomically Correct Testbed (ACT) hand, [21] investigates how tendon coordination patterns influence the positional precision of the hand. The decrease in precision is measured after tying various tendons to the same actuator. A small reduction in actuators is possible without large penalties on the fingertip precision. The metamorphic hand [22]-[24] also relies on the kinematic analysis of the hand movements in order to improve the overall design. The main objective is to assess the effects of a reconfigurable palm to the overall hand functionality. The methodology is mainly focused on hands rather different to the human, but it provides some basic principles that can be considered in the anthropomorphic hand design. Finally, the authors in [1] assess how different types of robotic hand components affect the tradeoff between robustness to clutter and grasp stability.

In the prosthetic hand design, being able to control dexterous hands with only a few signals is crucial due to the limited bandwidth of human-hand interfaces. The design of those interfaces is the major bottleneck in prosthetic hands nowadays [25], and none of the proposed methods have been proved to be sufficiently reliable for commercial applications [26]. In addition, hand weight and reliability are key factors of user acceptance [27].

In summary, determining the quality of a hand design and assessing the effect of different parameters on the resulting functionality is difficult and remains an open problem. The work presented in this paper provides a methodology for comparison as well as a publicly available benchmark data. In the following section, we provide the details of the proposed methodology.

\section{SySTEM DESCRIPTION AND METHODOLOGY}

We first define the term action manifold $\mathcal{A}$ that represents all the postures (or a chosen subset) that a hand can reach. For example, we may generate an action manifold that represents all three-fingered grasps for a hand. To compare different hand kinematic designs, we generate different actions manifolds, both for human and artificial hands. We define a hand configuration via the specification of its fingertip poses in the corresponding fingertip space. Although the dimensionality of the representation space is rather high, the actual dimensionality of the fingertip data is lower since it occupies only a small portion of the representation space. The objective is then to use the action manifold, represented in fingertip space, for defining a metric for evaluation of similarity between different hands.

We present the basic idea of the approach in Fig. 1. The figure shows a hypothetical visualization of the fingertip space, and the idea of its use for assessing the similarity between three hands.

Let us assume that "Hand 2" represents the space populated by typical human hand motions, while "Hand 1" and "Hand 3 " represent the motions generated by two other five-fingered kinematic hand structures. The aim of our approach is to estimate the intersection between the volumes spanned by two hands, i.e., to estimate which postures both hands are capable of achieving. In the case of comparing a human and an artificial hand, the degree of overlap can reveal the level of anthropomorphism of the artificial hand. This overlap is expressed via the AI. In 


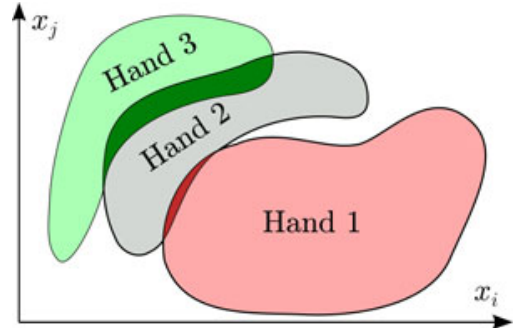

Fig. 1. Hypothetical visualization of the fingertip space and the embedded action manifolds. Depending on the kinematic structure of the hand, the shape of the action manifold differs.

summary, the comparison is addressing primarily the kinematic behavior of the hands.

Comparing the occupancy space associated with different hands is difficult as we do not know the actual density of the data, but only have access to point estimates. Thus, we need to model the associated density of the data corresponding to each hand. Comparing such high-dimensional data is computationally expensive, but we can exploit the lower dimensionality of the action manifold when modeling the density. To that end, we use a probabilistic dimensionality reduction approach, which finds a parameterization of the density approximation using a single low-dimensional latent variable.

\section{A. System Overview}

The first step is to generate an action manifold for a human hand. To make the comparison and visualization feasible, the manifold spanned by the human hand motion is projected onto a lower dimensional space. This projection is performed using a nonlinear dimensionality reduction. All possible fingertip configurations of an artificial hand are then projected onto that low-dimensional space. One example of this mapping is shown in Fig. 2 as step 4. Overall, the system consists of the following steps shown in Fig. 2.

1) Human data generation: First, a dataset of human grasping movements is generated. These movements define the benchmark action manifold for further comparison. Details are presented in Section V-A.

2) Dimensionality reduction: Nonlinear dimensionality reduction is used to project the high-dimensional manifold to a lower dimensional space suitable for visualization and comparison. Details are given in Section IV-A.

3) Artificial hand dataset: Similar to the human dataset, a dataset of the artificial hand movements is generated based on its forward kinematics.

4) Projection: This dataset is projected onto the lowdimensional space spanned by the human data. The projection of artificial hands is presented in Section VII.

5) Comparison: The overlap between the manifolds is evaluated in the lower dimensional space. In order to quantify the overlap, we created an overlap measure, i.e., the AI, as explained in Section V-D.

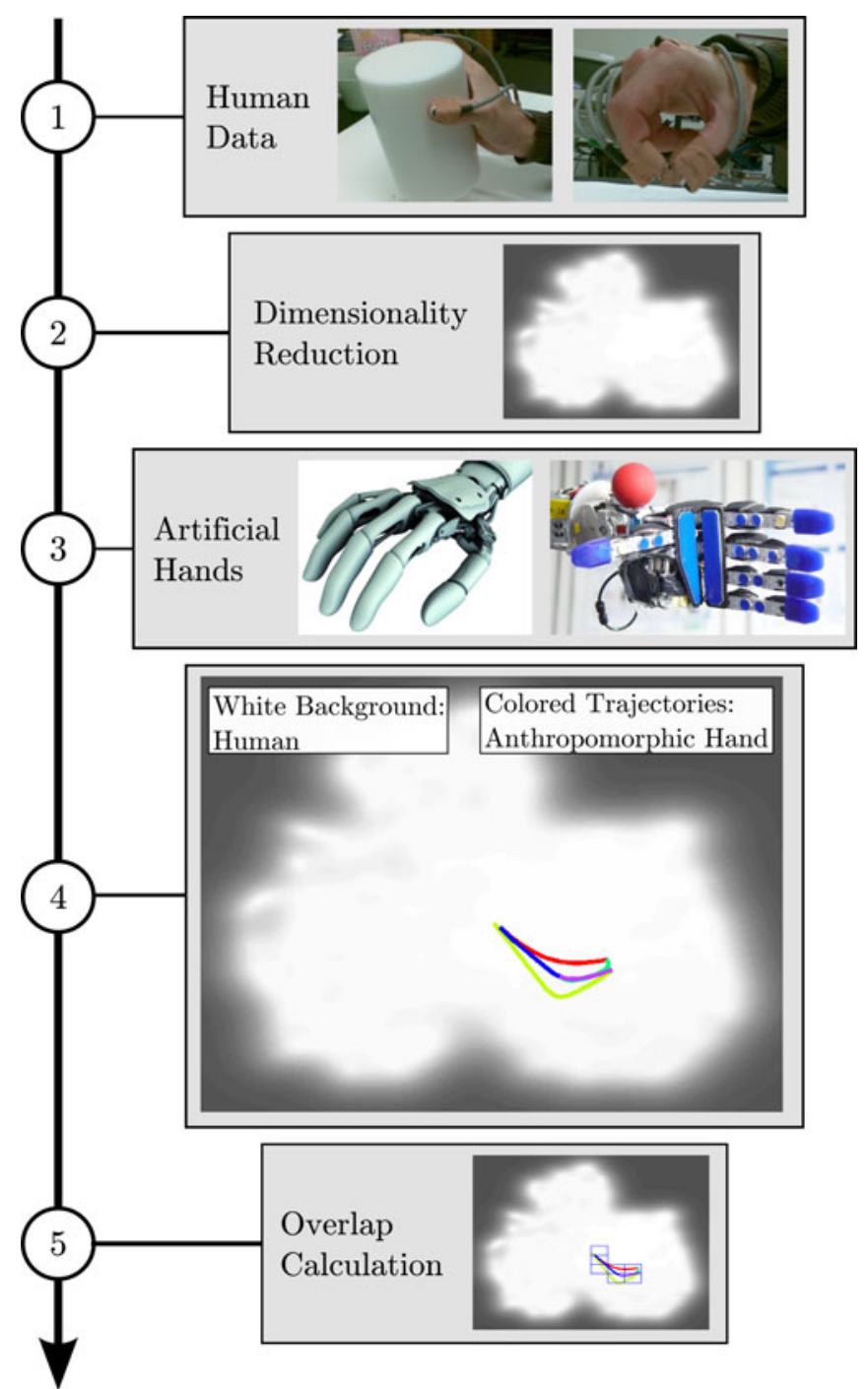

Fig. 2. System overview: The recorded human hand movements (1) are projected onto a 2-D space using nonlinear dimensionality reduction (2). The movements of an artificial hand (3) are then projected to that space (4), and the overlap is used as the basis for comparison (5).

\section{Nonlinear Dimensionality RedUCtion}

To obtain the lower dimensional representation for comparison, we make use of state-of-the-art nonlinear dimensionality reduction techniques. The first reason for the choice of these techniques stems from the fact that the hand data are highly nonlinear. The second reason is that these techniques, as it will be discussed in the next section, provide the possibility of not only encoding the data in a lower dimension but to provide a likelihood measure as well. Finally, the employed technique gives us the possibility of encoding the high-dimensional data in a compact low-dimensional manner suitable for the comparison.

\section{A. Gaussian Process Latent Variable Models (GP-LVM)}

The technique we employ is Gaussian process latent variable model (GP-LVM), which is a generative dimensionality reduction model. Let $D$ denote the dimension of the data space and $q$ the dimension of the low-dimensional latent space. Given $N$ 
observations in the fingertip space $\mathbf{T}$, the matrix containing the data points is denoted $\mathbf{Y} \in \mathbb{R}^{N \times D}$, and the matrix of the corresponding points in the latent space is $\mathbf{X} \in \mathbb{R}^{N \times q}$. The $i$ th observation in the fingertip space is denoted as $\mathbf{y}_{i} \in \mathbb{R}^{1 \times D}$ and in the latent space $\mathbf{x}_{i} \in \mathbb{R}^{1 \times q}$, respectively. Assuming that the observed data have been generated through a functional mapping with additive Gaussian noise

$$
\mathbf{y}_{i}=f\left(\mathbf{x}_{i}\right)+\epsilon
$$

where $\epsilon \sim \mathcal{N}\left(\mathbf{0}, \sigma^{-2} \mathbf{I}\right)$, the likelihood $P(\mathbf{Y} \mid \mathbf{f})$ of the data can be formulated. The underlying idea of the model is to place a Gaussian process $(\mathcal{G P})$ prior over the generative mapping $f$. Combining this with the likelihood and integrating out the mapping leads to the marginal likelihood of the data

$$
P(\mathbf{Y} \mid \mathbf{X}, \theta)=\prod_{j=1}^{D} \frac{1}{(2 \pi)^{\frac{N}{2}}|\mathbf{K}|^{\frac{1}{2}}} e^{-\frac{1}{2} \overline{\mathbf{y}}_{j}^{T} \mathbf{K}^{-1} \overline{\mathbf{y}}_{j}}
$$

where $\overline{\mathbf{y}}_{j} \in \mathbb{R}^{N \times 1}$ is the $j$ th column of the data matrix $\mathbf{Y}$. The probability is calculated as the product of $D$ independent Gaussian processes, each responsible for one dimension of the data space. The covariance matrix $\mathbf{K}$ defines the notion of similarity between points $\mathbf{x}_{i}$ and $\mathbf{x}_{j}$, and is constructed using a kernel function with the hyperparameters $\theta$. Here, $\mathbf{K}$ takes the form of an RBF (radial basis functions) kernel combined with bias and white noise terms

$$
k\left(\mathbf{x}_{i}, \mathbf{x}_{j}\right)=e^{-\frac{\gamma}{2}\left(\mathbf{x}_{i}-\mathbf{x}_{j}\right)^{T}\left(\mathbf{x}_{i}-\mathbf{x}_{j}\right)}+\sigma_{b}+\sigma_{n} \delta_{i j} .
$$

The solution to the latent locations and the hyperparameters of $\mathbf{K}$ can be found by iteratively maximizing (2).

Back Constraints: In its basic form, the GP-LVM does not guarantee the existence of a smooth inverse to the generative mapping [28]. However, this can be incorporated into the model by representing the latent locations $\mathbf{x}_{i}$ in terms of a smooth parametric mapping $g_{j}$ from the observed data $\mathbf{y}_{i}$ :

$$
x_{i j}=g_{j}\left(\mathbf{y}_{i}, a\right)=\sum_{n=1}^{N} a_{j n} k_{b c}\left(\mathbf{y}_{i}, \mathbf{y}_{n}\right)
$$

where $k_{b c}$ is the back constraint kernel. This implies that the maximum likelihood solution of the parameters $a$ rather than the latent locations are sought. This is referred to as a backconstrained GP-LVM [28]. In addition to constraining the latent location to preserve the local smoothness of the observed data, previously unseen data can be projected onto the latent space in an efficient manner by pushing them through this back mapping. We use an RBF kernel of the following form:

$$
k\left(\mathbf{y}_{i}, \mathbf{y}_{j}\right)=e^{-\frac{\gamma}{2}\left(\mathbf{y}_{i}-\mathbf{y}_{j}\right)^{T}\left(\mathbf{y}_{i}-\mathbf{y}_{j}\right)}
$$

where the inverse kernel width $\gamma$ controls the smoothness of the function. When projecting previously unseen points to the latent space, a sum over all contributions of the points from the training data is calculated.

\section{B. Representation of Rotation}

The data involve 3-D position and orientation of the fingertips. This data will be interpreted as high-dimensional vectors

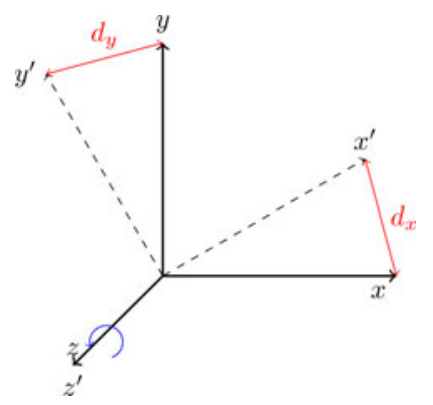

Fig. 3. Euclidean distance between rotation matrices $R$ and $R^{\prime}$ interpreted as 9 -D vectors. The vectors correspond to the directions defined by the columns of the rotation matrices. An orientation $R$ is rotated $30^{\circ}$ around its $z$-axis to obtain $R^{\prime}$. The corresponding distance between the orientations is the Euclidean norm of a vector composed by the distances $d_{x}, d_{y}$, and $d_{z}$ between each of the axis normal vectors.

and compared in an Euclidean way by GP-LVM. While the representation of position data is straightforward, a representation of rotation in which the Euclidean norm reflects similarity is less obvious. We shortly review the different ways of representing the rotation.

Euler angles employ only three parameters. The main drawback is the nonsmoothness of the representation and the existence of the singularities (gimbal lock) [29]. Thus, comparing directly the Euler angles as 3-D vectors is not appropriate.

Quaternions use four parameters, and the representation is compact, smooth and has no singularities [29]. The main drawback is that the Euclidean distance does not always reflect the similarity. Namely, the signs of the components of the quaternion can be inverted without affecting the transformation matrix [30, p. 162]. Therefore, the quaternion $\mathbf{q}=\left(e_{0}, e_{1}, e_{2}, e_{3}\right)$ represents the same rotation as $\mathbf{q}^{\prime}=-\mathbf{q}=\left(-e_{0},-e_{1},-e_{2},-e_{3}\right)$. The Euclidean distance between such a pair of quaternions is $\left\|\mathbf{q}-\mathbf{q}^{\prime}\right\|=\|2 \mathbf{q}\|=2$, as quaternions are normalized $\|\mathbf{q}\|=1$.

Rotation matrices use a $3 \times 3$ matrix, which uniquely defines the orientation of an object at the cost of introducing additional dimensions. The columns of the rotation matrix can be seen as points whose position vectors correspond to an axis of the rotated system (see Fig. 3). This means that the Euclidean distance of their displacement varies smoothly with that of the orientation, implying that the representation encodes the similarity we seek. By concatenating the rotation matrix $\mathbf{R}$ as a point $\mathbf{r} \in \mathbb{R}^{9 \times 1}$, the Euclidean norm of the corresponding vector will encode how similar two rotations are

$$
\begin{aligned}
\mathbf{R} & =\left(\begin{array}{lll}
x_{0} & y_{0} & z_{0} \\
x_{1} & y_{1} & z_{1} \\
x_{2} & y_{2} & z_{2}
\end{array}\right) \\
\mathbf{r} & =\left(x_{0}, x_{1}, x_{2}, y_{0}, y_{1}, y_{2}, z_{0}, z_{1}, z_{2}\right)^{T} \\
\left\|\mathbf{r}-\mathbf{r}^{\prime}\right\| & =\left(\sum_{i=0}^{2}\left(x_{i}-x_{i}^{\prime}\right)^{2}+\sum_{i=0}^{2}\left(y_{i}-y_{i}^{\prime}\right)^{2}+\sum_{i=0}^{2}\left(z_{i}-z_{i}^{\prime}\right)^{2}\right)^{\frac{1}{2}} \\
& =\left(d_{x}^{2}+d_{y}^{2}+d_{z}^{2}\right)^{\frac{1}{2}}
\end{aligned}
$$

Thus, only the rotation matrix suits our need. 


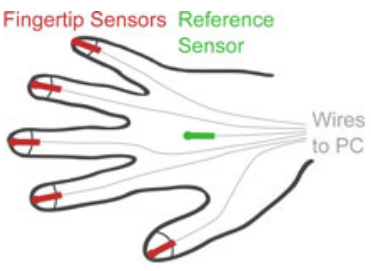

(a)

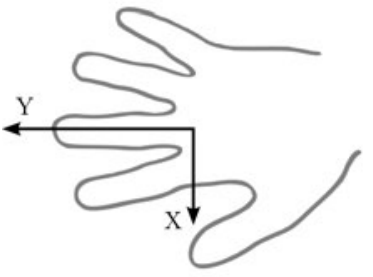

(c)

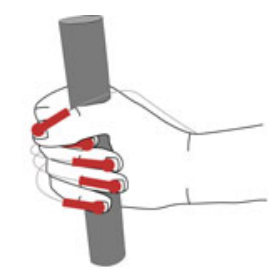

(b)

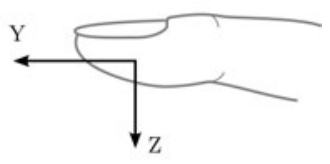

(d)
Fig. 4. Sensor placement and coordinate system definition for the recording of the human grasping data: (a) Sensors on the fingertips and the dorsum of the hand. (b) Example posture for grasp number 2. (c) and (d) Coordinate system definition for the reference and fingertip sensors. Both are aligned with the anatomical directions.

\section{Pose Representation}

As shown in the previous section, the Euclidean distance between the components of the rotation matrix effectively reflects similarity in orientation. However, in order to apply the GP-LVM approach, we need to compare, in a Euclidean manner, the different poses (position and orientation). To compare poses using an Euclidean norm, we need to make sure that the relative scale of each dimension corresponds to similar "distortions" in terms of pose. To that end, we independently transform the dimensions of the parameter space such that each possible configuration is contained within a hypercube. This has the implication that we consider a translation of the length of a hand to correspond to a rotation of $\pi$ radians. Smaller rotations and translations are scaled accordingly. By bounding the parameter space to a hypercube, we effectively encode an invariance to different hand sizes.

\section{DATA GENERATION AND ENCODING}

\section{A. Human Action Manifold}

To obtain a representative human action manifold, human grasping data were recorded. The focus was put on one-handed static grasps, based on measurements of five subjects (three males and two females). All subjects were right handed and did not report any hand disabilities. The average hand length and width were $185.2 \mathrm{~mm} \times 81.1 \mathrm{~mm}$, with standard deviations of 13.3 and $7.4 \mathrm{~mm}$. The hand measurements were in accordance with the protocol provided in [31]. A Polhemus Liberty system with six magnetic sensors was used to record the data. The spatial and angular resolution of each sensor is $0.8 \mathrm{~mm}$ and $0.15^{\circ}$, respectively. A sensor was applied to the nail of each fingertip. An additional sensor was placed on the dorsum of the hand as a reference. See Fig. 4(a) for how the markers were applied to the hand. The subjects were asked to perform 31 different grasp types described in [32] on an object typical of each action. Initially, the hand was placed flat on the table next to the object to be grasped. Upon a start signal, the subject had to grasp the object with the desired grasp type, lift the object [this moment is shown in Fig. 4(b)], replace it, and return the hand to the starting position.

The data recording started when the hand began to move and ended when the hand has returned to the initial position. Each grasp type was performed twice. The second trial is used for training and the first for testing (see Section VI-B). The fingertip sensors were transformed into the coordinate system of the reference sensor in order to remove global hand movement. The resulting dataset consists of 4650 data points (30 samples of each grasp trajectory $\times 31$ grasp types $\times 5$ subjects $)$. Each fingertip is described by a 12-D vector (three encoding positions, nine rotations). Focusing on five-fingered hands, the vector representing one hand configuration has $5 \times 12=60$ elements. By selecting a different reference dataset, we can prioritize certain capabilities of the robot hand by deciding which actions/grasps are important. For example, if only very small objects are to be manipulated, then there may be no need to include big objects since it would promote hands with power grasp capabilities.

\section{B. Robotic Action Manifolds}

The action manifolds of the robotic hands are obtained via kinematic hand models implemented in MATLAB. The joint space of the hands is sampled and the corresponding fingertip configurations are determined. The scaling and dimensionality of the dataset is done in analogy to the human hand dataset- the positions are normalized by the hand length, and the orientations are transformed into rotation matrices. There is no strict rule on how dense the sampling of the joint space has to be, but there is a guideline. The inter-point distance of the latent space projection should be smaller than the discretization of the latent space (i.e., the box length). A further increase of the density of the sampling will not increase the AI as the boxes are already populated by at least one point.

\section{Low-Dimensional Space}

To create the low-dimensional space, we use the MATLAB FGPLVM toolbox [33]. We have previously shown that using the GP-LVM model, a 2-D space is sufficient to encode the variations in this data. To this end, we use a 2-D latent representation [12]. In addition this is beneficial as it provides simple means of generating intuitive visualizations. The appearance of the latent space can be quite diverse, depending on the scaling of the data and parameters of the GP-LVM. By systematic variation of the GP-LVM parameters, 50 models were created, and evaluated. As it will be presented in detail later, the models are compared in terms of their ability to distinguish between random hand models and the test set.

Regarding the human dataset, the subjects generate a similar trajectory when they perform the same grasp type, allowing for a calculation of the mean grasp trajectory. To gain an insight into the structure of the space, Fig. 5 presents five such trajectories. Depending on the grasp type, the final posture corresponds to a 


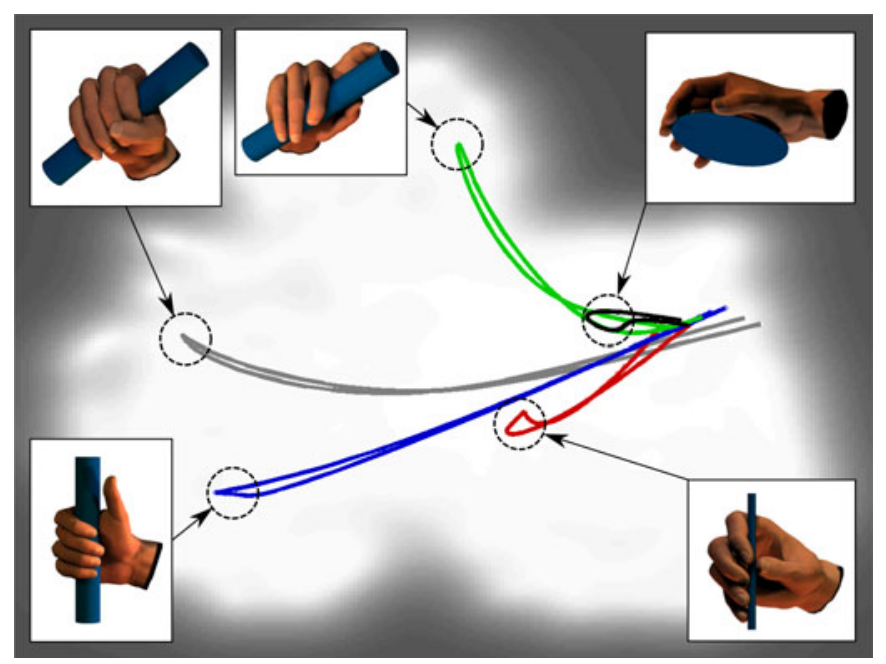

Fig. 5. Latent space representation of the grasping data. The trajectories correspond to the average trajectory of five subjects performing selected grasps. Each grasp type is located at a distinct area in latent space.

different location in the latent space. From the starting position (in the center on the right side of the latent space), the subjects proceed to the final grasping point (indicated by circles) and then return back to the start position. There is a relationship between the location in the latent space and the finger flexure. A grasp on the left size of the latent space tends to be a grasp where the fingers are flexed more. This trend is natural since the starting posture is a flat hand, and as the fingers flex, the difference to that posture increases, thus moving farther away from the starting position.

\section{Anthropomorphism Index}

Once the movements of the artificial hand are projected onto the latent space spanned by the human, the overlap between those trajectories and the human-spanned manifold has to be measured. Our approach is to discretize the latent space into a regular grid and count how many cells are populated by a given hand design.

An important parameter for the calculation is the width of the cells, as we regard all points within one cell as being equal. With equal we mean that if we vary the position within that margin, then the resulting hand posture will only change to a nonsignificant degree. As presented, each subject performed the grasp types twice. The difference in the final grasp posture of the hand of trial one and trial two can be regarded as being irrelevant since both configurations resulted in a stable grasp. Points belonging to the actual grasping poses of trial one and two are projected onto the latent space, and the distance between two corresponding points is averaged over all trials and subjects. This gives a maximum distance $d_{x}$ and $d_{y}$ in $x$ - and $y$-direction, respectively, that can be regarded as being the same grasp. Those lengths will define the resolution of the grid in latent space.

The GP-LVM models the mapping from the latent to highdimensional space using a Gaussian process. This mapping provides us a mean (prediction of the high-dimensional location of the point) and a variance. The inverse of the variance, or confidence, is related to how certain the model is when reconstructing that point. The confidence $c$ is scaled into the interval $[0,1]$, where the white area in the latent space plots corresponds to maximal confidence. In regions, where there are many data points, the variance of the projection will be very low. Consequently, the confidence will be close to 1 . In sparse regions, the confidence will fall off as the projection gets more uncertain. A measure of the area of the human spanned latent space $a_{h}$ can be calculated by summing the area of each cell $a_{b}=d_{x} \cdot d_{y}$ weighted by their corresponding confidence $c_{i}$ as $a_{h}=\sum_{i} c_{i} \cdot a_{b}$. The projection of the artificial hand movements discretized into $m$ steps will result in a set of points $\mathbf{p} \in \mathbb{R}^{M \times 2}$ whose overlap $a_{r}$ will be calculated. This is done by summing over all cells $b_{i}$, which are populated by at least one point $\mathbf{p}_{k}$

$$
a_{r}=\sum_{i} a_{b} \cdot \begin{cases}c_{i}, & \exists \mathbf{p}_{k} \in b_{i} \\ 0, & \text { otherwise. }\end{cases}
$$

Finally $a_{r}$ can be set into a relation to the area of human spanned space $a_{h}$ and the relative latent overlap can be calculated. The ratio ${ }^{a_{r}} /_{a_{h}}$ is multiplied by 100 to obtain a percentage value. We refer to this value as AI. It shows what percentage of the human dataset is covered by the robotic hand:

$$
\mathrm{AI}=\frac{a_{r}}{a_{h}} \cdot 100
$$

\section{Evaluation OF THE LATENT SPACE}

We seek a latent space, where the overlap between a human and a nonanthropomorphic hand is minimal. In GP-LVM, we can use different parameters to influence the structure of the latent space. One of these parameters is the inverse width of the back constraints kernel $\gamma$. As described in Section IV-A, the projection from high to low-dimensional space is governed by back constraints. In (5), we can observe that the ratio between the distance between points $\left\|\mathbf{y}_{i}-\mathbf{y}_{n}\right\|$ and the inverse width $\frac{1}{\gamma}$ determines the influence of different high-dimensional points $\mathbf{y}_{n}$ on the low-dimensional point $\mathbf{x}_{i}$.

Fig. 6(a) represents a situation in which the kernel width is small compared with the interpoint distances. In this case, the contribution of any external point becomes negligible. New anthropomorphic data (white circles) will not be supported by our latent representation in this case; thus:

$$
\frac{1}{\gamma} \ll\left\|\mathbf{y}_{i}-\mathbf{y}_{n}\right\| \Rightarrow a_{j n} e^{-\frac{\gamma}{2}\left(\mathbf{y}_{i}-\mathbf{y}_{n}\right)^{T}\left(\mathbf{y}_{i}-\mathbf{y}_{n}\right)} \approx 0, n \neq i .
$$

A large kernel width makes all points in the original space to equally support any point in the latent space [see Fig. 6(c)], no matter if they are anthropomorphic (white circles) or not (crosses); thus:

$$
\frac{1}{\gamma} \gg\left\|\mathbf{y}_{i}-\mathbf{y}_{n}\right\| \Rightarrow a_{j n} e^{-\frac{\gamma}{2}\left(\mathbf{y}_{i}-\mathbf{y}_{n}\right)^{T}\left(\mathbf{y}_{i}-\mathbf{y}_{n}\right)} \approx 1, \forall n .
$$

Our goal is to use a value of $\gamma$ such that only those points that correspond to anthropomorphic postures are taken into account [see Fig. 6(b)]. As we cannot directly determine how well the kernel width represents the manifold, we ensure that the chosen 


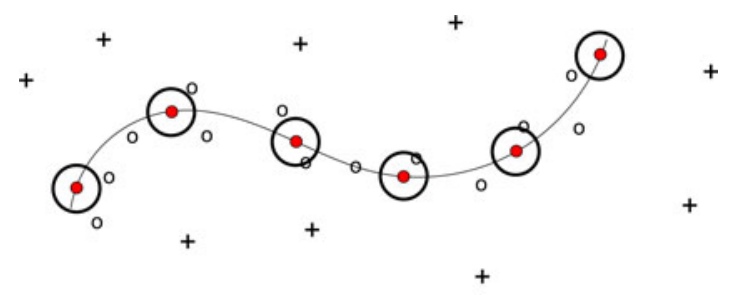

(a)

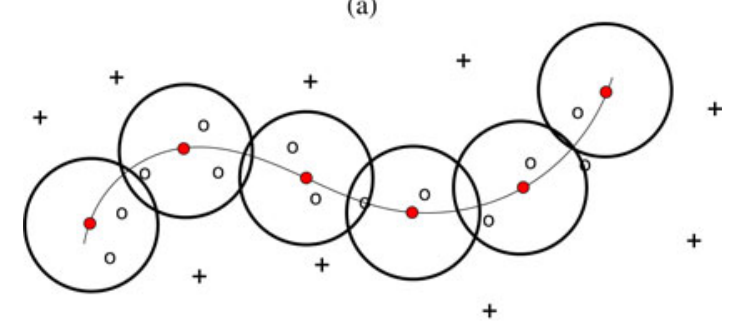

(b)

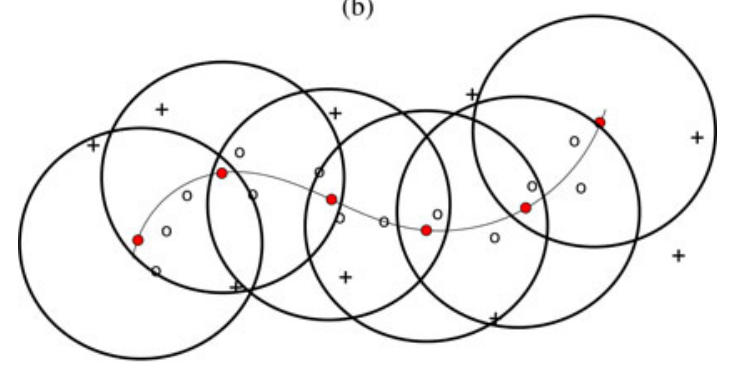

(c)

Fig. 6. Influence of kernel width on the discrimination between the test set (empty dots) and the random hand set (crosses). The manifold (indicated by the line) is sampled using data points from the training set (filled circles) and their corresponding kernel width (circles). (a) Manifold is not represented properly as the kernel width is too small to incorporate the training data. (b) Kernel width supports the inclusion of the training data and exclusion of the random data. (c) Kernel width is large and includes noninformative data.

width results in random models obtaining a very low AI, while human grasping data obtain a very high AI.

\section{A. Random Models}

We want the resulting space to have the ability to discriminate between human-like and nonhuman-like hands. Thus, when the movements of a nonanthropomorphic hand are projected onto the latent space, spanned by the human hand movements, the AI should be minimal. This should occur even in the case of high-dimensional nonanthropomorphic hand data, which might have a large action manifold.

To test the behavior when projecting nonanthropomorphic hand data, we create multiple random hand models. The random models are created using random Denavit-Hartenberg parameters with $3 \mathrm{DoF}$ for each finger. Additionally, the positions and orientations of the bases of the fingers are random; the relative orientation of the fingertip coordinate frame to the kinematic chain is random as well. Joint angles are selected randomly from a 15-dimensional uniform distribution between 0 and $2 \pi$. Overall, we take 20000 random samples from the joint space and calculate the corresponding fingertip poses. To model the

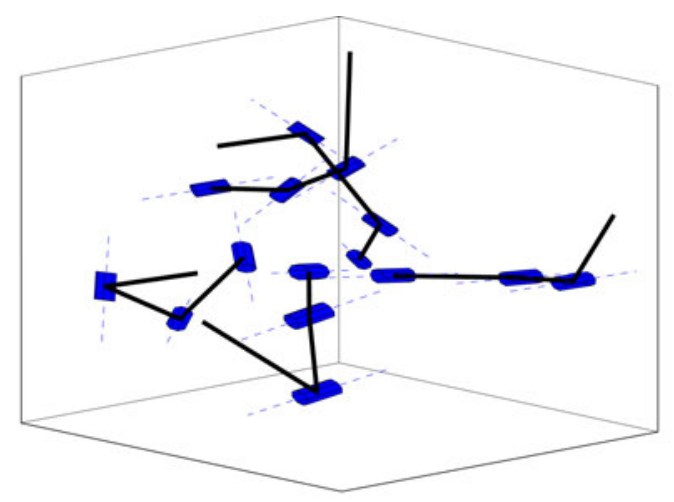

(a)

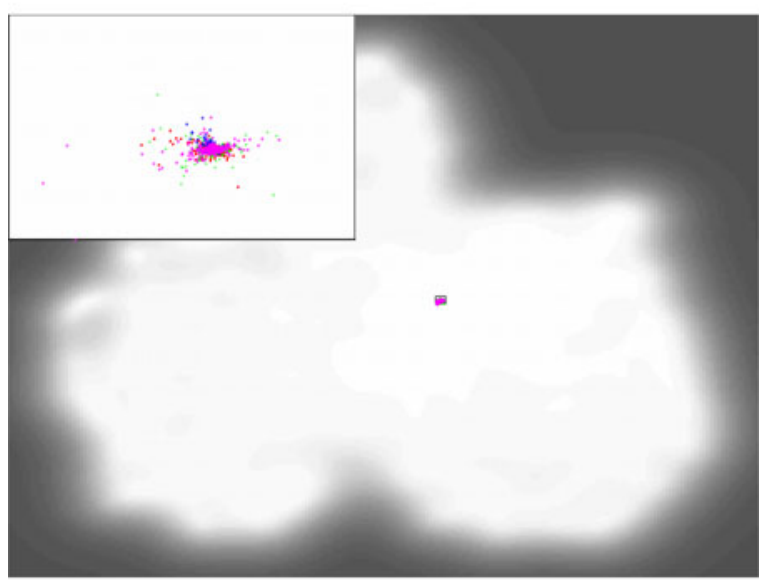

(b)

Fig. 7. If a random hand (a) is projected onto the latent space, then it covers only a very small area of the human manifold (b). (a) A random hand model: Five kinematic chains represent the fingers of the hand. (b) Projection of four random hand models to the latent space, indicated by different colors. Magnified in the top left corner.

hands, we use a MATLAB robotic toolbox [34], which allows us to calculate the fingertip poses through forward kinematics.

Fig. 7(a) shows a typical representative of the set of random hand models. By inspection, it becomes clear that this hand setup is not anthropomorphic. If we project the movements of four such random hands to the latent space [see Fig. 7(b)], then we see that all the movements collapse into a very limited region in the middle of the latent space. Hence, it is shown that the AI of hands, which are nonanthropomorphic, is close to zero. The model has the first desired property - artificial hands that are different from the human do not have a large AI score.

\section{B. Test Set}

The aim of this section is to show that, given the correct hand postures, all of the latent space can be covered with data points. In order to verify this, we project the test set to the latent space. The test set is similar to the training set as in both cases the subjects succeeded in grasping the object with the demanded grasp type. The only difference between the sets is the variation introduced by the executions of the users. In Fig. 8, the projection of the test set is shown. We observe that the test points are scattered accordingly with the training set. Yet, due to 


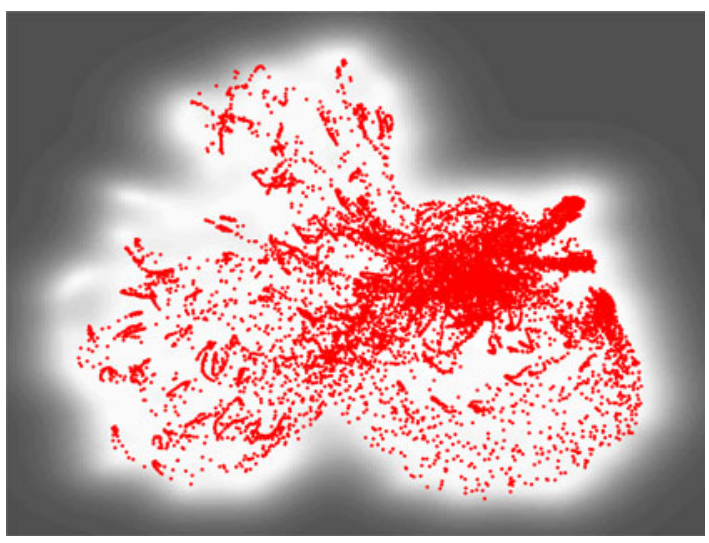

Fig. 8. Projection of the test set data to the latent space; the points cover most of the human spanned manifold (i.e., white area).

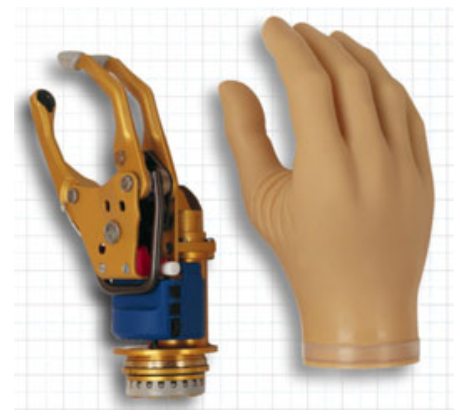

Fig. 9. The Otto Bock SensorHand ${ }^{\circledR}$ Speed. (Left) The hand without the covering glove, and (right) the glove that is put over the hand for protection of the hand and for cosmetic reasons.

the width of the kernel, there is a "halo" around the points, which increases the area $A_{h}$, and thus, the AI score of the training set is $67 \%$. This sets an upper bound for the maximal AI score that artificial hands can achieve.

\section{EXPERIMENTAL EVALUATION}

In this section, we evaluate the proposed methodology on two prosthetic and one robotic hand. A short discussion is provided for each hand, both regarding the data generation and comparison with the human hand.

\section{A. Sensor Hand}

The Otto Bock SensorHand ${ }^{\circledR}$ Speed (SHS) [35] is a prosthetic hand (see Fig. 9) with three actuated fingers, which are all driven by the same motor. The mechanical structure is covered by a glove, which is responsible for protecting the mechanics of the hand and creating a more human-like appearance. The glove also emulates the ring and the little finger, resulting in a fivedigit design. There is a metal bar within the glove which couples fingers four and five to the movements of the middle finger. As they are solely connected via the glove, the movement amplitude decreases from middle to little finger. The forward kinematics of the hand takes that into account by reducing the maximal finger flexion of the ring and little finger. The finger angles $\alpha_{i}$, where $i=1$ is the thumb, and $i=5$ is the little finger, depend

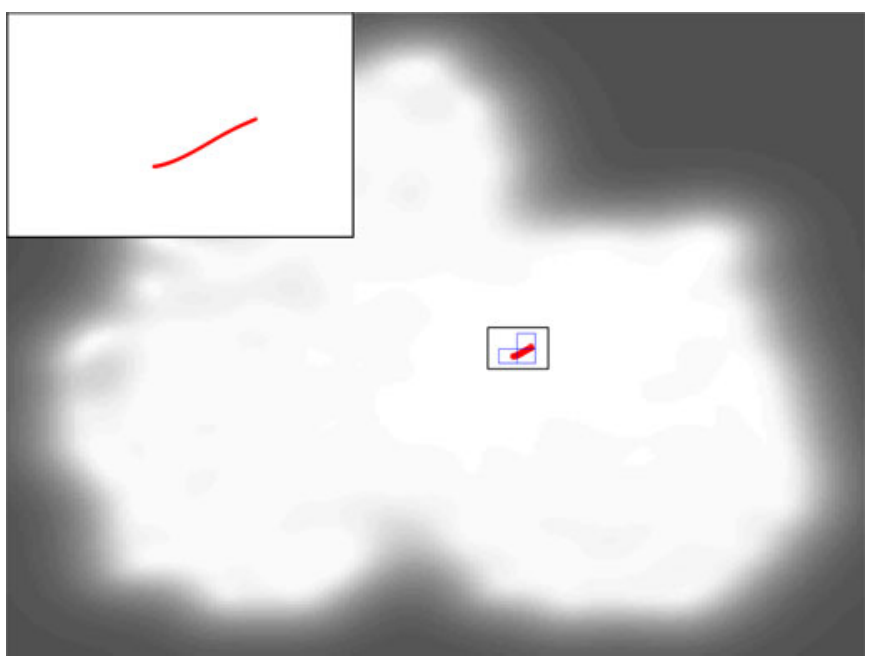

Fig. 10. Projection of the fingertip movements of the Otto Bock SensorHand to the latent space. The red points represent the trajectory of one open-close cycle. The hand has an AI of $0.25 \%$.

on the driving variable $c$, where $c=43^{\circ}$ is hand closed, and $c=0^{\circ}$ is hand opened. Overall 100 equally spaced samples of $a$ were taken from that range. The corresponding finger flexion angles $\alpha_{i}$ are as follows: $\alpha_{1,2,3}=c, \alpha_{4}=0.9 \cdot c$, and $\alpha_{5}=0.8 \cdot c$. Fingertip poses are computed based on these flexion angles. Their projection during one opening-closing cycle, which is all the hand is capable of, is shown in Fig. 10. The hands' AI is $0.25 \%$. The trajectory is different to the projection of a random hand [see Fig. 7(b)].

Overall, the SHS has some major differences from a human hand. The position of the thumb is not anatomically correct; it is basically rotated $180^{\circ}$ so that it perfectly opposes the index and middle finger. Even though the positions of the thumb fingertip are potentially correct, the orientations are not. The human cannot orient the fingertip in such a way as the SensorHand does. Additionally, all finger $\mathrm{MCP}^{2}$ joints share the same rotation axis. A more natural way would be to orient the axes in such a way that the fingers are slightly abducted when the MCP joint is extended. All those non-anthropomorphic features combined are the reason why the latent space trajectory of the hand is relatively short.

As we use hand models, the properties of the hand setup can be changed easily, and the effect on the latent space overlap can be analyzed. As an example, we actuate the five joints independently, which are originally coupled in the SHS (the $\mathrm{CMC}^{3}$ of the thumb and the MCP joints of the fingers), conferring five DoFs to the hand. The range of motion is the same as before, and we take nine equally spaced flexion values for each joint. Overall this creates $9^{5}=59049$ different hand postures, which are projected to the latent space. The projection (see Fig. 11) shows that increasing the dimensionality of the hand does not change the latent space overlap much. The much more complex hand has an $\mathrm{AI}$ of only $0.4 \%$, which is a slight increase to the

\footnotetext{
${ }^{2}$ The metacarpophalangeal joint connects the metacarpus to the first phalanges (fingers) in the human hand.

${ }^{3}$ The carpometacarpal joint is the most proximal joint of the human thumb.
} 


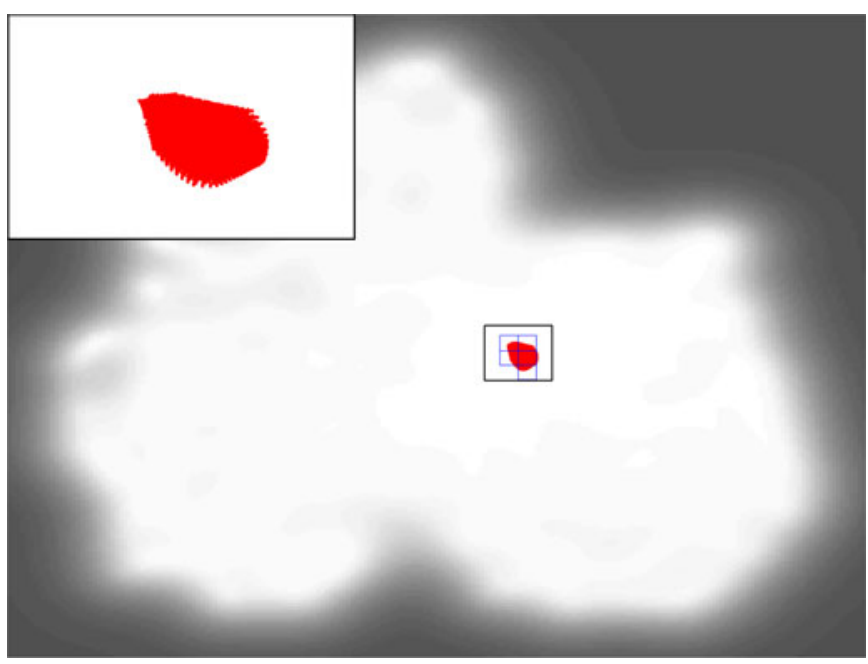

Fig. 11. Projection of the fingertip movements of the 5 DoF "Otto Bock SensorHand" onto the latent space. The hand has an AI of $0.4 \%$.

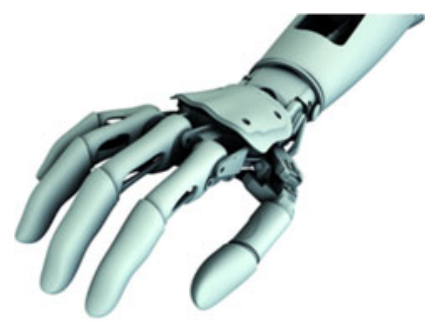

Fig. 12. Otto Bock Michelangelo ${ }^{\circledR}$ hand [36].

original SHS. Adding independent actuators proved to be a bad choice for increasing the hand's anthropomorphism.

\section{B. Michelangelo Hand}

The next generation of prosthetic hands by Otto Bock is the Michelangelo ${ }^{\circledR}$ hand [36] (see Fig. 12), with 2 DoF. The axes of the finger MCP joints are oriented in a more natural way. The fingers are slightly abducted when the MCP joints of the fingers are extended, whereas when flexed, the fingertips adduct and touch each other. The first DoF is the main drive, which is responsible for a coordinated flexion and extension of the five digits. The second DoF changes the thumb position-it can be abducted or adducted. As the hand is still in development, the exact control scheme of the hand has yet to be finalized. Therefore, we used a current hand implementation that had the following hand postures: 1) hand open for tripod pinch (OT);2) hand open for lateral pinch (OL);3) neutral position (NP); 4) tripod pinch (TP); 5) lateral pinch (LP).

The following movement trajectories between positions are incorporated into the hand model: OT $\rightarrow \mathrm{TP}, \mathrm{OL} \rightarrow \mathrm{LP}, \mathrm{NP}$ $\rightarrow \mathrm{OT}, \mathrm{NP} \rightarrow \mathrm{OL}, \mathrm{NP} \rightarrow \mathrm{TP}$. Each trajectory is sampled with 100 points, and the corresponding fingertip poses are projected onto the latent space. Fig. 13 shows the projection of these movements, where the colors indicate different trajectories. Compared with the SensorHand, it can be observed that the trajectories are much longer. Therefore, the hand achieves a

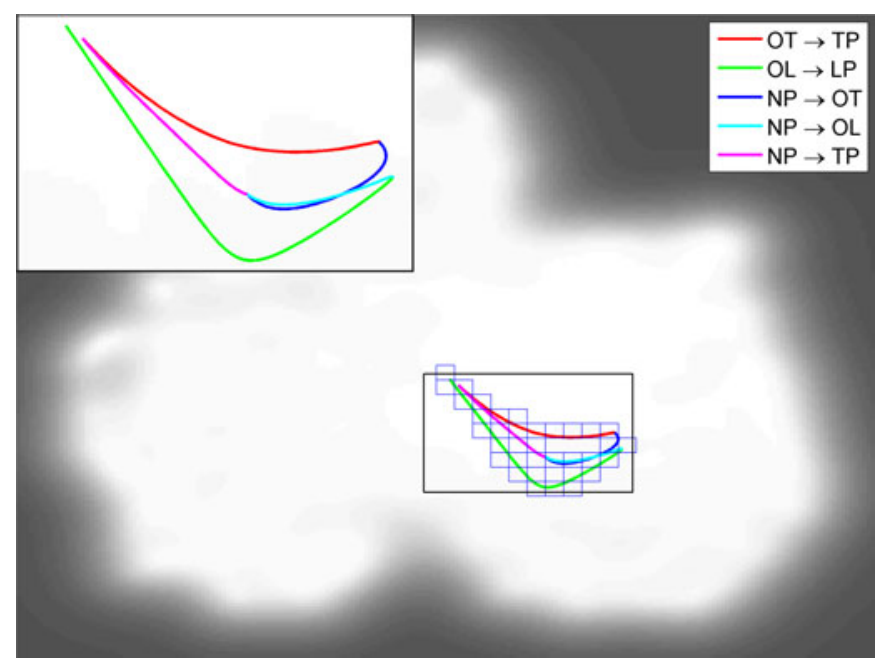

Fig. 13. Projection of the fingertip movements of the Otto Bock Michelangelo Hand to the latent space. The AI of the hand is $2.8 \%$.

larger AI of 2.8\%. Even though the hand has very few DoFs, its score is significantly larger compared with the SensorHand speed.

The TP and LP are located on the left-hand side of Fig. 13, whereas the hand is opened on the right side of the latent space. In between is the NP with trajectories connecting it to OT, OL, and TP. If the movements of the Michelangelo hand are compared with the human grasp trajectories of Fig. 5, then it can be observed that they also show a left-right dominance and that the starting position is on the right side, whereas the grasp positions are on the left. That can be regarded as a sign that not only the hand is capable of covering larger areas in the human manifold but that the movements themselves are human like as well. The positions of the tripod pinch and the lateral pinch are relatively close in latent space. That is due to the system roughly weighting every finger the same. As the poses of four of the five digits are nearly identical (in the lateral pinch the fingers flex a little bit more), it is plausible that the projections in the latent space are similar.

As already demonstrated with the SensorHand, we theoretically increased the complexity of the hand by assigning the hand $5 \mathrm{DoF}$ - the flexion of each digit is actuated independently. As the thumb has an additional DoF (abduction/adduction), this value had to be specified as well. It was set into the intermediate rest position. Changing the value of this joint does not affect the results to a large degree.

The range of motion from each digit is sampled with nine angles, resulting in the same number of points as in the SHS case. The resulting AI is $7.9 \%$, which is considerably more than the $2.8 \%$ the original hand has. Thus, introducing additional DoFs is a suitable way to equip the hand with more kinematic capabilities that resemble the human hand. If we observe the projection of the 5 DoF Michelangelo in Fig. 13, then we see that the extreme position in the right-left direction corresponds to the open and the grasp position, respectively. All movements of the original Michelangelo lie beneath the line connecting those two positions and have very roughly a triangle shape. The 


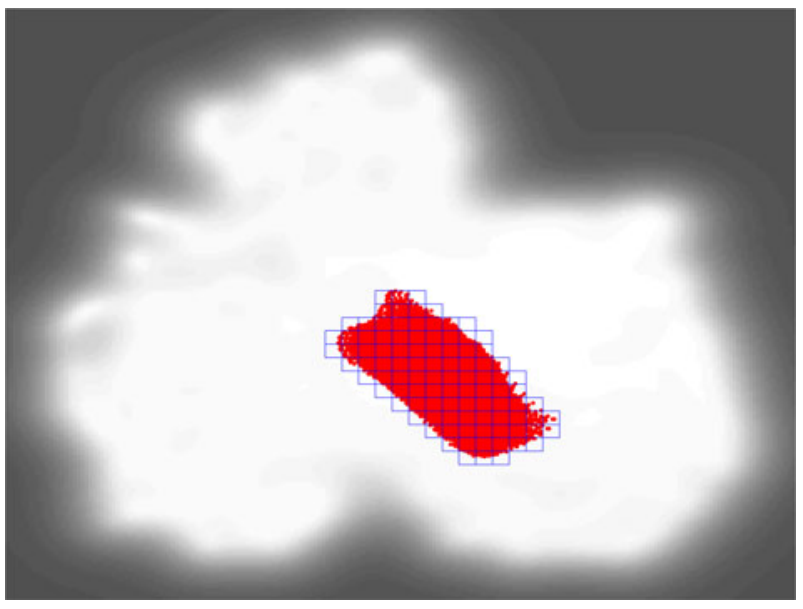

Fig. 14. Projection of the virtual 5-DoF Michelangelo hand to the latent space. The red points represent the area the hand can reach, and it results in an AI of $7.9 \%$.

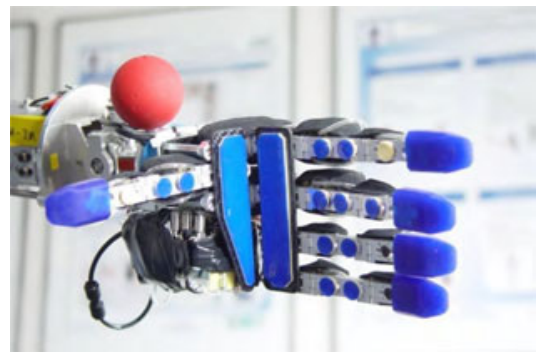

Fig. 15. FRH-4 hand [37].

movements of the 5-DoF hand overlap an additional space above that triangle. The top point in the projection in Fig. 14 corresponds to a hand position, where the index finger is extended, but the other fingers are flexed, and the thumb is in moderate flexion. The ability to individually flex fingers is important to reach new areas in the latent space. That is a difference with the SensorHand, where the introduction of finger individualization does not influence substantially the latent space overlap.

\section{FRH-4 Hand}

As an example of a hand with many independent DoFs, we use the FRH-4 hand [37] built for the mobile-assisting robot ARMAR. With eight independent fluidic actuators, it has a much more complex actuation system than the two prosthetic hands described in the previous sections. Its general appearance (see Fig. 15) is supposed to be human like; it has a size that is comparable with the human hand, and the kinematic setup has some similarities. One design goal of the hand was to be anthropomorphic, but another goal was to develop a hand which is suitable for robotic grasping. To meet the second design objective, a tradeoff on the anthropomorphism had to be accepted. One major difference is in the palm setup - the FRH-4 hand has one DoF in the metacarpus, which allows the palm to flex in the middle. The human hand does not share this as the palm is rigid in the longitudinal direction. Fig. 15 shows the palm joint in a flexed position, whereas in the extended position the fingers

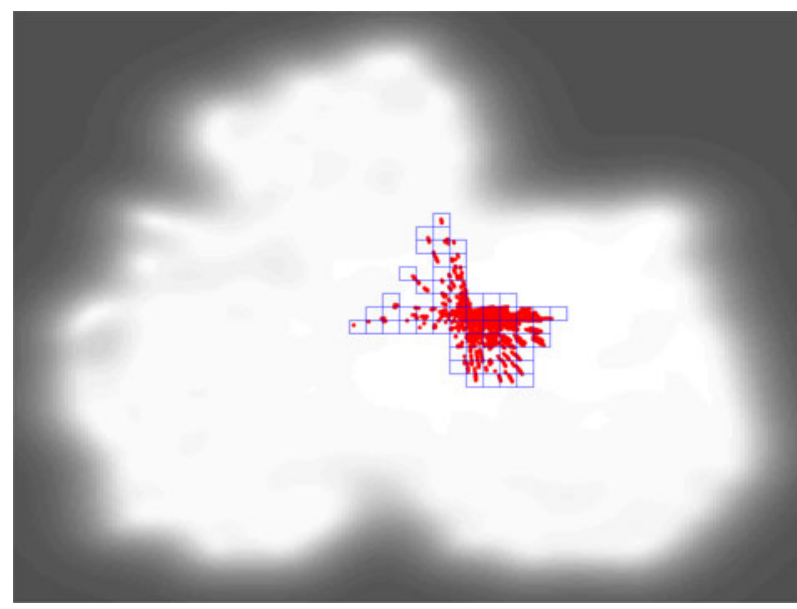

Fig. 16. Projection of the FRH-4 hand. It has an AI of 5.2\%.

would point to the upper right corner of the picture. The index and the middle finger both have $2 \mathrm{DoF}$, one joint represents the MCP joint of the human, and the other one is in between the proximal interphalangeal (PIP) and distal interphalangeal (DIP) joints. The ring and little fingers have one combined DoF, that is, a common flexion in the MCP joint. All joint axes of the fingers are parallel and the finger segment lengths are $40 \mathrm{~mm}$. The thumb has two actuators, which actuate the CMC joint and the joint between the MCP and IP (interphalangeal) joint of the thumb. The base of the thumb is exactly opposing the index and the middle fingers. This setup is very similar to the SensorHand and substantially different from the human hand, where the axes of the thumb are not aligned with the axes of the fingers.

Each of the eight DoFs has a range of $90^{\circ}$, and to calculate all hand configurations, we took four samples from each of the joint workspaces. Each joint can be flexed by $\left\{0^{\circ}, 30^{\circ}, 60^{\circ}\right.$, $\left.90^{\circ}\right\}$, and due to the high dimensionality of the hand, this leads to a total number of $4^{8}=65536$ hand configurations. Further increasing the number of samples would require prohibitively large computational times.

As the kinematic structure makes it difficult to define where the hand length could be measured (which is used for scaling the positions prior to projecting to the latent space), we performed a parameter sweep through all possible hand lengths and then determined the hand length with the maximal overlap. This length was assumed to be the correct hand length, and the results corresponding to that length are given. The resulting hand length of the FRH-4 hand is $25 \mathrm{~cm}$, which is larger than the maximal human hand length of about $21.15 \mathrm{~cm}$ [31]. The width of the FRH-4 hand is $9.3 \mathrm{~cm}$ [37], which is comparable with the hand width of a large human hand [31]. The calculated hand length is slightly too large given the hand width, but due to the different kinematic setup to the human hand, that difference is acceptable. For the prosthetic hands presented in the paper, the hand length did not have to be calculated, as there is information on the size available.

The projection of the hand with the determined hand length of $25 \mathrm{~cm}$ is shown in Fig. 16. The AI is 5.2\%. Compared with the large number of actuators, this is a relatively low value, given 
that the Michelangelo hand with only 2 DoF already has an AI of $2.8 \%$. As described above, the hand has some features which are not anthropomorphic, which explains the reduced score. In Fig. 16, we observe that the outermost points are slightly isolated from the rest. In order for the method to work correctly, the interpoint distance between neighboring points should be smaller than the box size. That is not the case here. Consequently, the sampling is not dense enough; thus, potentially only a few points are located at the intersection with the human action manifold. If we had increased the number of hand configurations, then we would have been able to further increase the overlap as those points would no longer be isolated. To test how much larger the overlap could be, we exchanged how to obtain the joint values. Instead of a systematic variation of the joint angles, we sampled the joint space with 60000 random points and calculated the corresponding overlap for five such sets. The result was an AI of $9.2 \pm 0.25 \%$. The different sampling method increased the overlap but is still small when compared with the human hand. For the SensorHand and the Michelangelo Hand, this resampling was not necessary as their joint space could be sampled densely enough.

\section{DISCUSSION AND CONCLUSIONS}

An important goal in the design of new robotics and prosthetic hands is to achieve high dexterity with minimum actuation. In this paper, we have presented a methodology for measuring the similarity between human and artificial hand motion capabilities. The similarity is determined by the definition of the AI. We concentrated on evaluating the capability of the artificial hands to produce human-like grasping motions. The evaluation is made based on human hand motion data generated with five test subjects. Similarly, artificial hands data are generated by sampling the joint space and calculating the corresponding fingertip poses via forward kinematics. The main contribution of our study is the development of a metric for performing the comparison based on state-of-the-art methods for nonlinear dimensionality reduction.

One advantage of our approach is that it offers flexibility with respect to the hands that can be tested. The hands can have an arbitrary kinematic structure and the joint couplings can be complex. The method can easily be used for other similar purposes: It only requires to generate a new underlying dataset. For example, if one wants to emphasize precision grasps, then one could record humans grasping a variety of small objects. The AI evaluates an important aspect of the hand capabilities-its kinematics. There are also other parameters that are of relevance such as speed, precision, and force. Further inclusion of the hand dynamic capabilities may provide a more complete analysis.

There are lessons learned in terms of the employed methodology. As the interpoint distances on the human manifold have a certain average value, the kernel width has to compensate for this. If this is not taken into account, then the manifold cannot be represented properly as there may be holes where the projection is not supported by data. This defines a minimal kernel length which can represent the manifold properly, as well as introduces a minimal selectivity perpendicular to the manifold. The sam- pling rate of the human dataset can be increased in order to improve the selectivity but at the cost of higher computational complexity. As there are no objects involved in assessing the anthropomorphic hand structures, passive compliance and underactuated hands cannot be implemented directly. Interaction with an object is needed to determine how the fingers wrap around it. In order to analyze hands with passive compliance, a workaround can be used by sampling the passive joints as well.

The experimental evaluation shows that hands with as little as two actuators (like the Michelangelo hand) are able to populate significant proportions of the latent space of the lowdimensional human hand movements. Several studies [9], [38] have shown that human hand movements can be described in a linear subspace of fewer than eight dimensions. According to those studies, having a hand with eight DoFs or less should be sufficient to cover most of the human hand movement.

In general, the tested hands covered an area with an overlap of less than $10 \%$; thus, the hands had significant limitations compared with the human hand. Some of the hands are not able to fully extend and flex the fingers due to rigid fingers (SensorHand and Michelangelo), having joint axes that are not well aligned with the movement axis of the human hand (SensorHand and FRH-4 Hand) or having a range of motion in the joints that is lower than the humans (SensorHand and Michelangelo). Those are the reasons for the reduced overlap, and the goal for the future is to overcome these limitations with as little effort (actuators, joints, etc.) as possible.

In the future, we will perform parameter studies to determine the influence of design parameters on the AI. This will provide insights into the relationship between kinematic elements and their influence on motion capabilities. The final goal is not only to change the parameters but to find the optimal kinematic structure with respect to the proposed AI.

\section{REFERENCES}

[1] M. T. Mason, S. Srinivasa, A. S. Vazquez, and A. Rodriguez, "Generality and simple hands," Robotics Inst., Carnegie Mellon Univ., Pittsburgh, PA, Tech. Rep. CMU-RI-TR-10-40, Nov. 2010.

[2] L. Biagiotti, F. Lotti, C. Melchiorri, and G. Vassura, "How far is the human hand? A review on anthropomorphic end effectors," DIES Internal Rep., Univ. Bologna, Bologna, Italy, Tech. Rep., 2004.

[3] M. C. Carrozza, G. Cappiello, S. Micera, B. B. Edin, L. Beccai, and C. Cipriani, "Design of a cybernetic hand for perception and action," Biol. Cybern., vol. 95, no. 6, pp. 629-644, Dec. 2006.

[4] C. S. Lovchik and M. A. Diftler, "The robonaut hand: a dexterous robot hand for space," in Proc. IEEE Int. Conf. Robot. Autom., vol. 2, 1999, pp. 907-912.

[5] H. Liu, K. Wu, P. Meusel, N. Seitz, G. Hirzinger, M. H. Jin, Y. W. Liu, S. W. Fan, T. Lan, and Z. P. Chen, "Multisensory five-finger dexterous hand: The DLR/HIT hand II," in Proc. IEEE/RSJ Int. Conf. Intell. Robots Syst., Sep. 2008, pp. 3692-3697.

[6] H. Rijpkema and M. Girard, "Computer animation of knowledge-based human grasping," SIGGRAPH, vol. 25, no. 4, pp. 339-348, 1991.

[7] B. Buchholz and T. J. Armstrong, "A kinematic model of the human hand to evaluate its prehensile capabilities," J. Biomech., vol. 25, no. 2, pp. 149-162, 1992.

[8] D. Dragulescu, V. Perdereau, M. Drouin, L. Ungureanu, and K. Menyhardt, "3D active workspace of human hand anatomical model," BioMed. Eng. Online, vol. 6, no. 1, pp. 1-12, 2007.

[9] M. Santello, M. Flanders, and J. F. Soechting, "Postural hand synergies for tool use," J. Neurosci., vol. 18, no. 23, pp. 10 105-10 115, Dec. 1998. 
[10] C. R. Mason, J. E. Gomez, and T. J. Ebner, "Hand synergies during reachto-grasp," J. Neurophysiol., vol. 86, no. 6, pp. 2896-2910, Dec. 2001.

[11] I. V. Grinyagin, E. V. Biryukova, and M. A. Maier, "Kinematic and dynamic synergies of human precision-grip movements," J. Neurophysiol., vol. 94, no. 4, pp. 2284-2294, 2005.

[12] J. Romero, T. Feix, H. Kjellström, and D. Kragic, "Spatio-temporal modeling of grasping actions," in Proc. IEEE/RSJ Int. Conf. Intell. Robots Syst., Oct. 2010, pp. 2103-2108.

[13] F. Lotti, P. Tiezzi, G. Vassura, L. Biagiotti, G. Palli, and C. Melchiorri, "Development of UB hand 3: Early results," in Proc. IEEE Int. Conf. Robot. Autom., 2005, pp. 4488-4493.

[14] A. Bicchi, "Revisiting grasping basics," presented at the Robot., Sci. Syst. Conf. VI: Workshop Grasp Acquisit.: How Realize Good Grasps, Zaragoza, Spain, Jun. 2010

[15] A. Bicchi, "Hands for dexterous manipulation and robust grasping: A difficult road toward simplicity," IEEE Trans. Robot. Autom., vol. 16, no. 6 , pp. $652-662$, Dec. 2000

[16] G. A. Kragten and J. L. Herder, "The ability of underactuated hands to grasp and hold objects," Mech. Mach. Theory, vol. 45, no. 3, pp. 408-425, Mar. 2010.

[17] M. Ciocarlie and P. Allen, "A design and analysis tool for underactuated compliant hands," in Proc. IEEE/RSJ Int. Conf. Intell. Robots Syst., Oct. 2009, pp. 5234-5239.

[18] M. Ciocarlie and P. Allen, "Data-driven optimization for underactuated robotic hands," in Proc. IEEE Int. Conf. Robot. Autom., May 2010, pp. 1292-1299.

[19] D. Pratichizzo, M. Malvezzi, and A. Bicchi, "On motion and force controllability of grasping hands with postural synergies," presented at the Robot.: Sci. Syst. Conf., Zaragoza, Spain, 2010.

[20] G. A. Kragten and J. L. Herder, "A platform for grasp performance assessment in compliant or underactuated hands," J. Mech. Design, vol. 132, no. 2, 2010.

[21] M. Malhotra and Y. Matsuoka, "The relationship between actuator reduction and controllability for a robotic hand," in Proc. EMBS Int. Conf. Biomed. Robot. Biomechatron., Sep. 2010, pp. 331-336.

[22] J. Dai, D. Wang, and L. Cui, "Orientation and workspace analysis of the multifingered metamorphic hand-Metahand," IEEE Trans. Robot. Autom., vol. 25, no. 4, pp. 942-947, Aug. 2009.

[23] L. Cui and J. S. Dai, "Posture, workspace, and manipulability of the metamorphic multifingered hand with an articulated palm," J. Mech. Robot., vol. 3, no. 2, p. 021001, 2011.

[24] G. Wei, J. S. Dai, S. Wang, and H. Luo, "Kinematic analysis and prototype of a metamorphic anthropomorphic hand with a reconfigurable palm," Int. J. Humanoid Robot., vol. 8, no. 3, pp. 459-479, 2011.

[25] W. Craelius, "The bionic man: Restoring mobility," Science, vol. 295, no. 5557, pp. 1018-1021, Feb. 2002

[26] P. Parker, K. Englehart, and B. Hudgins, "Myoelectric signal processing for control of powered limb prostheses," J. Electromyogr. Kinesiol., vol. 16 , no. 6 , pp. 541-548, Dec. 2006.

[27] P. J. Kyberd, C. Wartenberg, L. Sandsjö, S. Jönsson, D. Gow, J. Frid, C. Almström, and L. Sperling, "Survey of upper extremity prosthesis users in Sweden and the United Kingdom," J. Prosthetics Orthotics, vol. 19, no. 2, pp. 55-62, Apr. 2007.

[28] N. D. Lawrence and J. Quiñonero Candela, "Local distance preservation in the GP-LVM through back constraints," in Proc. Int. Conf. Mach. Learn., 2006, pp. 513-520.

[29] R. M. Murray, S. S. Sastry, and L. Zexiang, A Mathematical Introduction to Robotic Manipulation. Boca Raton, FL: CRC, 1994.

[30] P. E. Nikravesh, Computer-Aide Analysis of Mechanical Systems.. vol. 186, Englewood Cliffs, NJ: Prentice-Hall, 1988.

[31] J. W. Garrett, "Anthropometry of the hands of male air force flight personnel," Aerosp. Medical Res. Lab., Aerosp. Medical Div., Air Force Syst. Command, Wright-Patterson Air Force Base, OH, Tech. Rep. AMRL-TR69-42, 1970.

[32] T. Feix, R. Pawlik, H. Schmiedmayer, J. Romero, and D. Kragic. (2009, Jun.). "A comprehensive grasp taxonomy," in Robotics, Science and Systems: Workshop on Understanding the Human Hand for Advancing Robotic Manipulation. [Online]. Available: http://grasp.xief.net

[33] N. Lawrence, "Probabilistic nonlinear principal component analysis with gaussian process latent variable models," J. Mach. Learn. Res., vol. 6, pp. 1783-1816, 2005

[34] P. Corke, "A robotics toolbox for MATLAB," IEEE Robot. Autom. Mag., vol. 3, no. 1, pp. 24-32, Mar. 1996.

[35] O. Bock. (2011). SensorHand speed. [Online]. Available: http://www. ottobock.com/cps/rde/xchg/ob_com_en/hs.xsl/3652.htm
[36] O. Bock. (2011). Otto Bock at the trade fair 2010 Leipzig. [Online]. Available: http://leipzig.ottobock.de/index.php?id=161\&no_cache $=$ $1 \& \mathrm{~L}=1$

[37] I. Gaiser, S. Schulz, A. Kargov, H. Klosek, A. Bierbaum, C. Pylatiuk, R. Oberle, T. Werner, T. Asfour, G. Bretthauer, and R. Dillmann, "A new anthropomorphic robotic hand," in Proc. IEEE-RAS Int. Conf. Humanoid Robots, Dec. 2008, pp. 418-422.

[38] J. Ingram, K. Körding, I. Howard, and D. Wolpert, "The statistics of natural hand movements," Exp. Brain Res., vol. 188, no. 2, pp. 223-236, Jun. 2008.

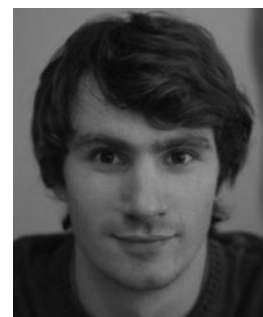

Thomas Feix received the M.Sc. degree in sports equipment technology from the University of Applied Sciences Technikum Wien, Vienna, Austria, and the Ph.D. degree from the Vienna University of Technology, in 2011.

$\mathrm{He}$ is a Postdoctoral Associate with the GRAB Lab, Department of Mechanical Engineering, Yale University, New Haven, CT. His research is focused on human grasping and manipulation and its application to robotics and prosthetics. He is working on human hand kinematics for manipulation.

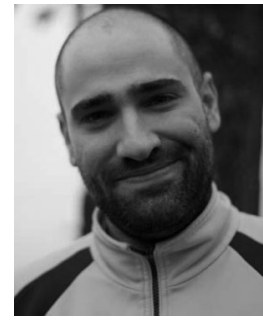

Javier Romero received the M.Sc. degree in telecommunication engineering from the Technical University of Madrid, Madrid, Spain, in 2007 and the Ph.D. degree in 2011 from the Royal Institute of Technology, Stockholm, Sweden.

$\mathrm{He}$ is a Postdoctoral Researcher with the Perceiving Systems Group, Max-Planck Institute for Intelligent Systems, Tübingen, Germany. His research interest is in robotic perception of humans and their environment, focusing on generative models of human appearance and human body tracking.

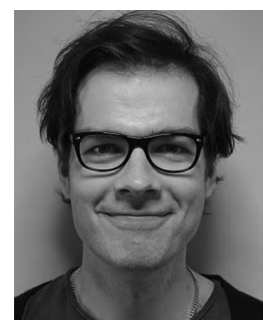

Carl Henrik Ek received the M.Sc. degree in vehicle engineering from Kungliga Tekniska högskolan (KTH), Stockholm, Sweden, in 2005 and the Ph.D degree in computer science from Oxford Brookes University, Oxford, U.K., in 2009.

$\mathrm{He}$ is a Postdoctoral Researcher with the School of Computer Science and Communication, KTH. His research areas include machine learning, robotics, and computer vision, specifically in probabilistic methods for learning efficient representations of data.

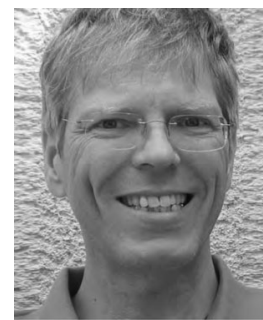

Heinz-Bodo Schmiedmayer received the M.Sc. degree in mechanical engineering in 1987 and the $\mathrm{Ph} . \mathrm{D}$. degree in engineering science in 1994, both from the Vienna Institute of Technology, Vienna, Austria.

$\mathrm{He}$ is an Associate Professor of biomechanics with the Institute of Mechanics and Mechatronics, Vienna University of Technology. His research is in the area of human movement science with a special focus on interaction with technical systems like prosthetics and sports equipment.

Dr. Schmiedmayer is a board member of the Austrian Society of Biomedical Engineering.

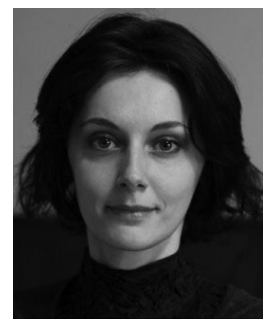

Danica Kragic received the M.Sc. degree in mechanical engineering from the Technical University of Rijeka, Rijeka, Croatia, in 1995 and the Ph.D. degree in computer science from Kungliga Tekniska högskolan (KTH), Stockholm, Sweden, in 2001

She is a Professor with the School of Computer Science and Communication at KTH. Her research areas include computer vision and robotics.

Dr. Kragic received the 2007 IEEE Robotics and Automation Society Early Academic Career Award. She is a member of the Swedish Royal Academy of Sciences and the Swedish Young Academy. 